\title{
Topographic Maps of Color Images
}

\author{
Bartomeu Coll \\ Dep. of Mathematics and Informatics \\ University of Illes Balears \\ 07071 Palma de Mallorca, Spain
}

\author{
Jacques Froment \\ PRISME, UFR Mathématiques et Informatique \\ Université Paris 5 R. Descartes \\ 45 rue des Saints-Pères, F-75270 Paris cedex 06
}

\begin{abstract}
We address the problem of extending topographic maps to color images. A topographic map gives a morphological and a geometrical representation of the information contained in natural images. Two approaches are presented and discussed. The first one is new and consists in defining a total order in $\mathbb{R}^{3}$ in accordance with the human visual perception of shapes. This allows to define color topographic maps in the same way that what it has been done for graylevel topographic maps. It has the advantage of leading all properties known in the gray-level case to remain true in the color case. But the map contains a so huge quantity of data that it has to be drastically simplified. The second approach, based on a so far unpublished result [4], allows to build a simplified representation by using the geometry given by the luminance component only. We present experiments which illustrate the advantages and the drawbacks of each method.
\end{abstract}

\section{Introduction and Background}

This paper discusses the extension of topographic maps to color images. Topographic maps of gray-level images have been recently introduced $[5,6]$ as a geometrical representation of the information contained in natural images. A lower (or upper) topographic map of a gray-level image $u: \Omega \subset \mathbb{R}^{2} \rightarrow \mathbb{R}$ is the family of the connected components of the lower (or upper) level sets of $u$, a lower level set $[u \leq \lambda]$ being the set of pixels $x$ such that $u(x)$ is lower than $\lambda$.

This representation leads to morphological edges [5, 9], which are selected pieces of level lines (level lines are border of level sets). Such structure better matches the perceptual edges properties than the classical Hildreth-Marr theory of edge detection : morphological edges are like perceptual edges invariant to local contrast changes, and they are connected curves. In addition, the topographic map may yield to a complete description (we just have to record the level $\lambda$ associated to each connected component) so that the original image $u$ may be reconstructed :

$$
u(x)=\inf _{\lambda}[u \leq \lambda]=\sup _{\lambda}[u \geq \lambda] .
$$

A scale parameter may be introduced to keep the most important morphological edges only, and from those a sketch image can be computed [8]. First applications of topographic maps and morphological edges include extraction of shapes [13, 10], comparison of images [3, 13], structured compression [9] and disocclusion [11] (recovery of hidden parts of an object occluded by another one).

The main drawback of this level set approach, which is probably accountable for the important delay between its introduction by the Mathematical Morphology school in the 1960s [14] and the use as an atomic representation, stands in the huge quantity of geometrical data associated to a topographic map : the amount of bytes needed to code a typical 8-bits $256 \times 256$ image by its topographic map is about 100 times greater than the amount obtained by the raw pixels description. Quite obviously, the applications based on topographic maps can't afford to deal with all of these data, and the representation must be widely simplified.

The right way to simplify a topographic map is to apply a morphological filtering on the gray-level image $u$. A filter $F$ is morphological if it is a contrast invariant operator [6], that is if, for any increasing continuous real function $g$, we have

$$
F(g(u))=g(F(u))
$$

The use of a morphological filter is justified by the property $[F(g(u)) \leq \lambda]=\left[F(u) \leq g^{-1}(\lambda)\right]$, which ensures that the family of level sets of the filtered image is invariant under any change of contrast. Classical examples of such contrast invariant operators are given by the Mathematical Morphology [14] : erosion, dilation, opening and closing. Another example is the anisotropic diffusion $F_{t}$ issued from the AMSS multiscale analysis [1]. It is possible to perform a morphological filtering by acting on the level sets : if $T$ is an increasing continuous operator on the closed sets family

()2000 IEEE. Published in the 2000 International Conference on Pattern Recognition (ICPR 2000), Barcelona, Spain, September 3-8, 2000.

Personal use of this material is permitted. However, permission to reprint/republish this material for advertising or promotional purposes or for creating new collective works for resale or redistribution to servers or lists, or to reuse any copyrighted component of this work in other works must be obtained from the IEEE. 
of $\mathbb{R}^{2}$, then the relation

$$
[F(u) \leq \lambda]=T([u \leq \lambda])
$$

defines a unique morphological filter $F$ [12]. A simple but powerful example of such operator is given by the grain filter [2] : it removes all "shapes" (levels sets with filled holes) having an area below a threshold. The grain filter is therefore useful in simplifying the topographic map, and if the threshold remains low it does not introduce visible degradation (it has been introduced to remove impulse noise).

Since we have $[g(u) \leq \lambda]=\left[u \leq g^{-1}(\lambda)\right]$, the family of level sets of $g(u)$ is equal to the family of level sets of $u$ : a contrast change preserves the topographic map. In [6] it is proved a stronger property : the topographic map of an image $u$ is equal to the topographic map of another image $v$ if and only if there exists a function $g(x, \lambda)$ increasing in $\lambda$ such that $v(x)=g(x, u(x))$ for all pixels $x$ (there are other technical properties on the function $g$, see [6] for details). Its means that $v$ is obtained from $u$ by applying a local contrast change. Topographic maps are therefore equivalence classes of images, modulo local contrast changes.

\section{Topographic Maps of Multivalued Images}

Let us now consider a color image, which is in the standard RGB color model a multivalued function $U=$ $(R, G, B): \Omega \subset \mathbb{R}^{2} \rightarrow \mathbb{R}^{3}$, where the three channels $R, G, B$ are the intensity of red, green and blue. What follows may be extended to functions with any number of channels. In this section, we address the problem of extending the topographic map definition and properties to such multivalued function $U$. Obviously, it would be nonsense to define the color topographic map as the family of the three topographic maps associated to each channel $R, G, B$ : there would be a lot of geometrical redundancy between each map, and a morphological filtering would be likely to introduce color artifacts. A better point of view is to switch to a color model which splits the channels between the intensity (also called luminance) and the chromaticity components. Indeed, natural gray-level images contain rich geometrical structures, which are far enough to reconstruct the shape of the objects. A color topographic map should therefore uses the luminance component first, with some refinements given by the chrominance.

Let $L(U), H(U)$ and $S(U)$ be the values of $U$ in the HSI color model ( $\mathrm{L}$ stands for Luminance or Intensity, $\mathrm{H}$ for Hue and $\mathrm{S}$ for Saturation). We define a total order $\preceq$ on $\mathbb{R}^{3}$ (an ordering relation is total if two elements are always comparable) by

$$
\begin{gathered}
U_{1}=\left(L_{1}, H_{1}, S_{1}\right) \preceq U_{2}=\left(L_{2}, H_{2}, S_{2}\right) \\
\mathbb{\Downarrow} \\
\left(L_{1}<L_{2}\right) \text { or }\left(L_{1}=L_{2} \text { and } H_{1}<H_{2}\right) \\
\operatorname{or}\left(L_{1}=L_{2} \text { and } H_{1}=H_{2} \text { and } S_{1} \leq S_{2}\right) .
\end{gathered}
$$

This is a lexicographic ordering relation, which has been applied alredy to process color images, see for instance [15]. We have chosen this order to fit the visual perception of geometrical structures : to detect shapes, human eyes are first sensitive to luminance, then to hue and at last to saturation.

As in the gray-level case, we define the lower (or upper) color topographic map of $U$ to be the family of the connected components of the lower (or upper) level sets of $U$, the lower level set of level $\lambda=\left(\lambda_{L}, \lambda_{H}, \lambda_{S}\right)$ being

$$
[U \preceq \lambda]=\{x / U(x) \preceq \lambda\} .
$$

A color filter $F$ is said to be morphological if it is a contrast invariant operator, that is if for any increasing continuous function $G: \mathbb{R}^{3} \rightarrow \mathbb{R}^{3}$, we have $F(G(U))=G(F(U))$. Since $\preceq$ is a total order, one can check that all properties state in the case of gray-level images are still valid here.

The approach to extend the topographic maps to color images by means of the lexicographic order (4) sounds therefore to be the most natural. However, the drawback of the huge quantity of data contained in the map is now so critical that the scheme may be simply unusable. Indeed, since the conversion from RGB to HSI enhances the channel dynamics, the quantity of data obtained for a 24 bits color map is more than $256^{2}$ times the quantity associated to 8 bits gray-level map ! The only solution is to drastically simplify the topographic map during its computation, using morphological filters.

\section{Another Morphological Filtering Model for Color Images}

In a forthcoming paper, V. Caselles, J.M. Morel and one of us [4] propose a different approach to define the topographic map of color images (see also [7]). Now, an operator $F: \mathbb{R}^{3} \rightarrow \mathbb{R}^{3}$ is said to be a morphological filter if for any $U$ and any

$$
G(U)=\frac{g(L(U))}{L(U)} U
$$

we have $F(G(U))=G(F(U))$. Notice that, since $\frac{g(L)}{L}$ may be not increasing in $\mathbb{R}^{3}$, a morphological filter in the sense given by Section 2 is not necessary a morphological filter in this new sense.

The reason of this new definition is given by the following result [4] : we suppose $L$ bounded and of bounded variation on $\Omega$, and $H, S$ Lebesgue-integrable on $\Omega$ (for a particular choice of $L, H, S$ explained in [4]). Let $\mathcal{A}_{\infty}$ be the $\sigma$-algebra generated by the connected components of the sections of $U$, i.e., the $\sigma$-algebra generated by the connected components of sets of the form $[c \leq L<d]$, for levels $c<d$ such that $[c \leq L<d]$ is of finite perimeter. 
Then, the equation

$$
F(U)=\left(\begin{array}{c}
L(U) \\
E\left(H(U) \mid \mathcal{A}_{\infty}\right) \\
E\left(S(U) \mid \mathcal{A}_{\infty}\right)
\end{array}\right)
$$

defines a morphological filter. This result says that the map constructed with the family of the connected components of the lower (or upper) level sets of $L(U)$, and with chromatic components set to be the average of the values of $H(U)$ and $S(U)$ in these regions, defines a new image $\mathrm{F}(\mathrm{U})$ where $\mathrm{F}$ is a morphological filter. The image $\mathrm{F}(\mathrm{U})$ has piecewise constant chromatic components $H, S$ on each connected component of the topographic map of $L$. In this way, this approach does not require to define the topographic map of color images. It maintains that we can simplify a color image by keeping the geometry associated to the luminance component only.

\section{Experiments and discussion}

The two representations of color images we have introduced must remain accurate while the quantity of information has to be reduced. The following color plate presents some experiments made on the baboon image (picture A, $256 \times 256$ pixels, 24-bits per pixel). Picture B and $\mathrm{C}$ illustrate the use of the morphological filter described in Section 3. In $\mathrm{B}$, the luminance $L(U)$ is quantized very precisely with 256 different levels. But the topographic map used to compute the chrominance is built with 7 levels of $L(U)$ only. On each region of this map, the chrominance components are averaged but not quantized. As a result, we get an image with rich geometrical information given by the luminance only. However, since the geometry of the hue channel may be slightly different, color artifacts may appear and we can see some of them in the border of the baboon's muzzle. The topographic map of this luminance channel (with 256 levels) contains 355000 level lines. The associated representation takes $35 \mathrm{Mb}$ (Megabytes). Picture $\mathrm{C}$ is the same than B but the topographic map is quantized with the number of levels (7) corresponding to the computation of the chrominance. Now, only 9000 level lines remain and the size of the representation is $1 \mathrm{Mb}$. This representation may become really light if we apply another morphological filter, as the grain filter. Picture D gives the reconstruction obtained with a color topographic map explained in Section 2. In order to get a computable topographic map, the L,H,S channels are strongly quantized (8 levels for L, 17 for $\mathrm{H}$ and 4 for $\mathrm{S}$ ). In spite of the fact that this image contains 544 different colors only, the result is quite fair and there is no color artifacts. The color topographic map has 276000 level lines and the parameters are chosen to get the same quantity of data than for image $\mathrm{B}(35 \mathrm{Mb})$, so that the visual quality of $\mathrm{B}$ and $\mathrm{D}$ may be directly compared. The use of the color topographic map seems to give better results, but if we want to reduce the amount of data, it is hardly possible to decrease the number of quantization levels. The picture at the bottom of the color plate shows the geometry of the color topographic map after a grain filter has been applied (20000 remaining level lines). However, the resolution of the picture is altered by such filtering, and a compact representation based on a color topographic map may be hard to attain without a process that removes the geometrical redundancy between the three channels.

\section{References}

[1] L. Alvarez, F. Guichard, P.-L. Lions, and J.-M. Morel. Axioms and fundamental equations of image processing. Arch. Rational Mechanics and Anal., 16(9):200-257, 1993.

[2] L. Ambrosio, V. Caselles, S. Masnou, and J.-M. Morel. Connected components of sets of finite perimeter and applications to image processing. Preprint, 1999.

[3] C. Ballester, E. Cubero-Castan, M. Gonzalez, and J.-M. Morel. Contrast invariant image intersection. Preprint.

[4] V. Caselles, B. Coll, and J.-M. Morel. Geometry and color in natural images. In preparation.

[5] V. Caselles, B. Coll, and J.-M. Morel. A kanizsa programme. In Progress in Nonlinear Differential Equs. and their Applications, pages 35-55, 1996.

[6] V. Caselles, B. Coll, and J.-M. Morel. Topographic maps and local contrast changes in natural images. Int. J. Comp. Vision, 33(1):5-27, 1999.

[7] A. Chambolle. Partial differential equations and image processing. In Proc. of ICIP'94, pages 16-20, 1994.

[8] J. Froment. A compact and multiscale image model based on level sets. In Scale-Space Theories in Computer Vision, pages 152-163. Lecture Notes in Computer Science 1682, 1999. Proc. of Sec. Int. Conf. Scale-Space'99.

[9] J. Froment. A functional analysis model for natural images permitting structured compression. ESAIM:COCV Control, Optimisation and Calculus of Variations, 4:473-495, 1999.

[10] F. Guichard and J.-P. Tarel. Curve finder combining perceptual grouping and a kalman like fitting. In Proc. of ICCV'99, pages 1003-1008, 1999.

[11] S. Masnou and J.-M. Morel. Level lines based disocclusion. In Proc. of ICIP'98, 1998.

[12] L. Moisan. Traitement numérique d'images et de films : équations aux dérivées partielles préservant forme et relief. $\mathrm{PhD}$ thesis, University of Paris-Dauphine, 1997.

[13] P. Monasse and F. Guichard. Scale-space from a level lines tree. In Scale-Space Theories in Computer Vision, pages 175-186. Lecture Notes in Computer Science 1682, 1999. Proc. of Sec. Int. Conf. Scale-Space'99.

[14] J. Serra. Image analysis and mathematical morphology. Academic Press, 1982.

[15] G. S. V. Caselles and D. Chung. Filters, vector morphology and coupled median filters, vector morphology and coupled pde's: Theoretical connections. To appear in Journal of Mathematical Imaging and Vision. 

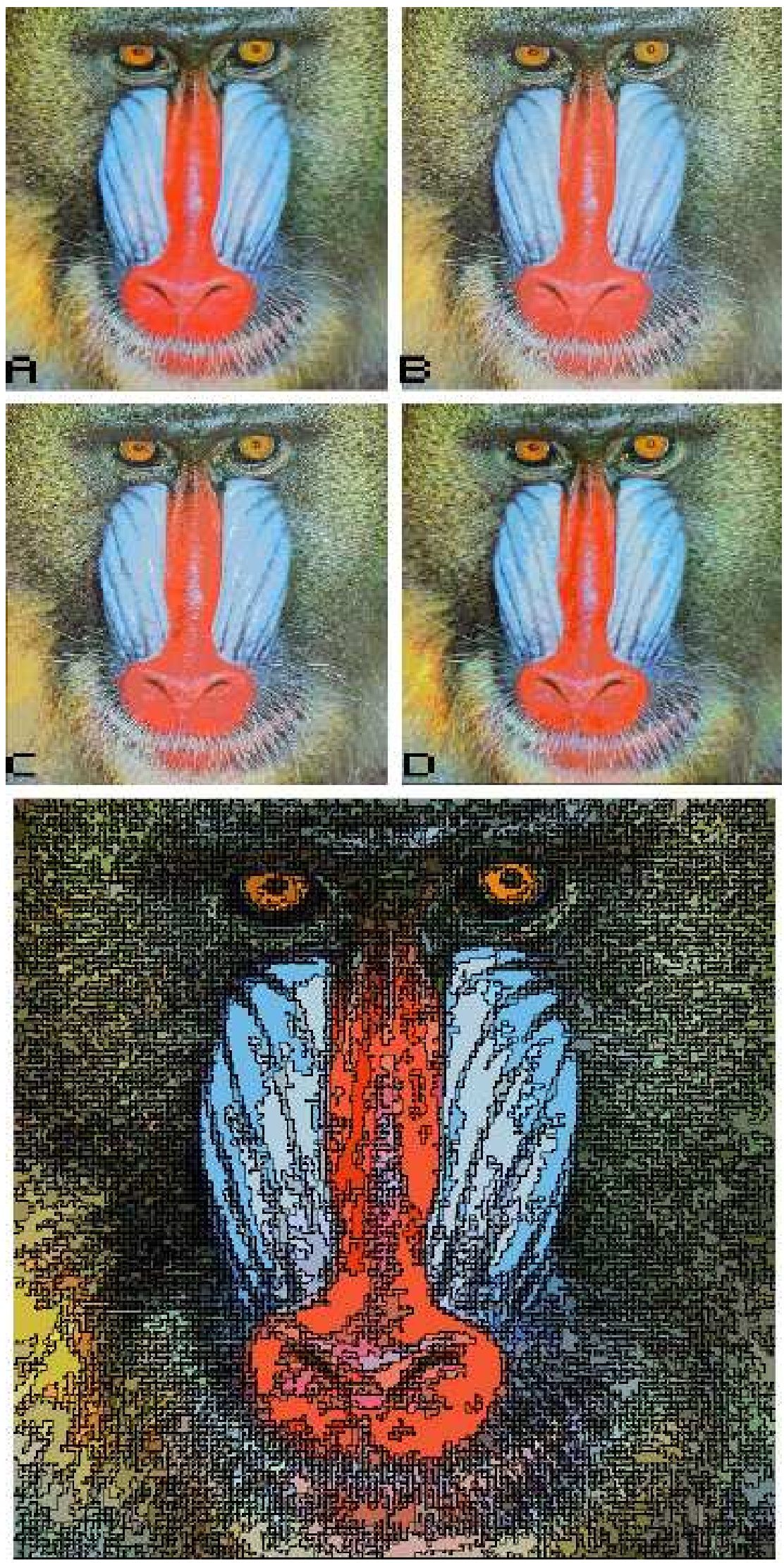